\title{
Cultura fiscal en los asalariados de Yucatán, México *
}

\author{
The Tax Culture of Salaried Employees in Yucatán, Mexico
}

\author{
Elisa Cabrera-Ignacio \\ Maestra en Administración Financiera, Universidad Tecnológica Metropolitana, \\ Mérida-México, elisa.cabrera@utmetropolitana.edu.mx
}

José Apolinar Zapata-Aguilar (D)

Doctor en Análisis Estratégico y Desarrollo Sustentable, Universidad Tecnológica Metropolitana, Mérida-México, jose.zapata@utmetropolitana.edu.mx

\begin{abstract}
Cómo citar / How to cite
Cabrera-Ignacio, E.; Zapata-Aguilar, J. A. (2021). Cultura fiscal en los asalariados de Yucatán, México. Revista CEA, v. 7, n. 14, e1653. https://doi.org/10.22430/24223182.1653
\end{abstract}

Recibido: 24 de diciembre de 2020

Aceptado: 22 de marzo de 2021

\section{Resumen}

Los impuestos representan el mayor porcentaje de los ingresos públicos de México; sin embargo, este es uno de los países con menor recaudación tributaria. Debido a ello, el presente artículo analizó la cultura fiscal de los municipios de Mérida, Umán y Tekax, en el Estado de Yucatán, México, tomando como muestra a un grupo de 267 trabajadores asalariados, con el objetivo de determinar si tienen la disposición de tributar al Estado de manera consciente, solidaria y responsable, basados en tres variables: creencias, actitudes y valores. Asimismo, teniendo en cuenta el salario mensual, se estudiaron las diferencias significativas en el nivel de cultura fiscal. Igualmente se realizó un estudio descriptivo-comparativo, donde se encontró que, en general, los trabajadores encuestados sí poseen una cultura fiscal que los dispone a tributar de manera consciente, solidaria y responsable al Estado. Al realizar el análisis, se observó una diferencia entre la cultura fiscal de los trabajadores con menores ingresos versus los de mayor ingreso, siendo los segundos, en contraste con los primeros, quienes tienen una cultura fiscal más propensa al cumplimiento del pago de impuestos. Además, se encontró que existe una relación positiva entre el nivel salarial y las creencias y valores relativos al pago de impuestos.

Palabras clave: cultura tributaria, individuos asalariados, recaudación tributaria, educación fiscal.

Clasificación JEL: D11, D13, D14.

*Este artículo se deriva del proyecto titulado «Cultura fiscal en los asalariados de Yucatán, México» y ha sido financiado con recursos propios. 


\title{
Highlights
}

- El nivel salarial de los empleados del Estado de Yucatán, México, tiene relación con la concepción de cultura fiscal.

- Existe una relación positiva entre el nivel salarial y la creencia de que es una obligación de los ciudadanos pagar impuestos que sirvan al Estado para costear servicios y obras públicas.

- En la medida que los niveles de salario incrementan, también incrementa la aceptación de que quien paga impuestos demuestra valores como la honradez, la responsabilidad y la solidaridad.

\begin{abstract}
Taxes represent the largest public income in Mexico; however, this country has one of the lowest tax collection rates. Therefore, this paper analyzes the tax culture in three municipalities in the State of Yucatán (i.e., Mérida, Umán, and Tekax) using a sample of 267 salaried employees. The aim of this study is to determine if they are willing to pay taxes in an aware, solidary, and responsible manner based on three variables: beliefs, attitudes, and values. Adopting a descriptive-comparative method, it was found that, in general, the surveyed employees do have a tax culture that disposes them to pay taxes in an aware, solidary, and responsible way. The analysis revealed a difference in tax culture between low- and high-income employees, i.e., the tax culture of the latter group makes them more prone to paying taxes. Furthermore, a positive relationship was found between salary level and beliefs and values related to paying taxes.
\end{abstract}

Keywords: Tax culture, salaried individuals, tax collection, tax education.

JEL classification: D11, D13, D14.

\section{Highlights}

- The salary level of employees in the State of Yucatán, Mexico, is related to their conception of tax culture.

- There is a positive relationship between salary level and the belief that citizens must pay taxes so that the government can fund public services and works.

- The higher the salary level, the more accepted is the idea that those who pay taxes demonstrate they have values such as honesty, responsibility, and solidarity.

\section{INTRODUCCIÓN}

De acuerdo con el Sistema de Información Legislativa en México (s.f.), los ingresos públicos se dividen en dos:

I) los ingresos ordinarios que son recaudados de forma regular por el Estado, tales como los impuestos, los derechos, los ingresos por la venta de bienes y servicios de los organismos y empresas paraestatales, etc; y, II) los ingresos extraordinarios, que son recursos que no se obtienen de manera regular por parte del Estado, tales como la enajenación de bienes nacionales, contratación de créditos externos e internos (empréstitos) o emisión de moneda por parte del Banco de México. 
Los impuestos representan el mayor porcentaje de ingresos públicos en México. De acuerdo con la Ley de Ingresos de la Federación para el Ejercicio Fiscal (2018), el ingreso total estimado para el ejercicio fiscal ese año fue de $\$ 5838$ 059.7, de los cuales $\$ 3311373.4$ correspondieron a impuestos, lo que representa el $56.72 \%$ del total.

De acuerdo con la Organización para la Cooperación y Desarrollo Económico (OCDE) y la Comisión Económica para América Latina y el Caribe ([CEPAL], 2020), México es uno de los países con menor recaudación tributaria como porcentaje del PIB. Para el 2018, la recaudación fue del $16.1 \%$, la cual estuvo por debajo del promedio de los países de América Latina y el Caribe (ALC), que estuvo alrededor de un $23.1 \%$, y aún más bajo con respecto al de la OCDE, el cual es del 34.3\%.

Lograr el cobro correcto y oportuno de los impuestos es una labor difícil para todos los gobiernos; sin embargo, hay países que realizan mejor esa tarea que otros (Abate, 2019; Huang, 2017). Vale la pena entonces analizar, bajo los siguientes interrogantes, los factores que podrían influir en la baja recaudación de impuestos que tiene México. ¿Saben los ciudadanos por qué se deben pagar impuestos? ¿Son conscientes que es a través del pago de impuestos que se aporta al Estado para costear los servicios públicos? ¿Tienen conocimiento sobre los impuestos? ¿Consideran un acto cívico el hecho de pagar impuestos?

En años recientes, la importancia de las consideraciones de la cultura fiscal (o cultura tributaria) (Richardson, 2008; Ermasova et al., 2021) se ha vuelta más evidente desde que las medidas necesarias para reformar los sistemas fiscales de economías en transformación han fracasado, en parte por su incompatibilidad con la cultura fiscal (Nerré, 2001) para que los países logren una administración exitosa de los impuestos. Por tal motivo, se ha requerido de la cooperación de los contribuyentes, en lugar de forzarlos a realizar cada aspecto de sus obligaciones fiscales (James et al., 2005).

La cultura fiscal se entiende aquí como el conjunto de valores, creencias y actitudes compartidos por una sociedad respecto a la tributación y las leyes que la rigen, lo que conduce al cumplimiento permanente de los deberes fiscales. Por lo tanto, el presente estudio tiene como objetivo determinar, a través de la aplicación de una encuesta dirigida, si la cultura fiscal de los trabajadores asalariados de Yucatán los incentiva a tributar al Estado de manera consciente, solidaria y responsable. Por su parte, los objetivos específicos aquí planteados buscan establecer las diferencias significativas en el nivel de cultura fiscal de los encuestados y determinar si existe relación entre el nivel salarial y las creencias, actitudes y valores que los estimulen a pagar impuestos.

\section{MARCO TEÓRICO}

\section{Antecedentes}

Desde la época prehispánica ha existido el tributo. De acuerdo con Amed y Negreiros (2000), como se citó en Domingues y González (2013), la palabra tributo «procede del latín tributum, -i, que a su vez proviene de tribu, - s, ciertamente una referencia a las tres primitivas tribus de Roma, señalándose que el verbo tributo, -ere significa dar, conceder». 
De acuerdo con la definición anterior, el tributo surge a partir de que el hombre se agrupa en tribus, es decir, cuando deja de ser nómada y se establece físicamente en algún lugar, y se da cuenta que requiere recursos que permitan atender las necesidades de la colectividad con la aportación de todos los habitantes. Lara Dorantes (2009) «los primeros núcleos sedentarios decidieron que, a fin de poder aprovechar plenamente las ventajas que este método de vida social ofrecía, era conveniente asignar el poder público ya constituido una nueva tarea: la satisfacción de las necesidades colectivas».

Para lograr la satisfacción de esas necesidades colectivas (Eagon, 2017), expone que se pensó que era justo que parte de los recursos o bienes que obtuvieran los miembros de la tribu fueran a parar a manos de sus líderes para sufragar el costo de los beneficios que recibe la comunidad (Lara Dorantes, 2009).

Ahora bien, a pesar de que el tributo nace como un concepto justo para contribuir a la sociedad y satisfacer las necesidades existentes, en la práctica se percibe más como una obligación más que como una aportación razonada; incluso, se puede llegar a sentir como a un acto coercitivo por parte del gobierno o autoridad estatal, lo cual se corrobora con la definición del SAT (2020) que indica que «los impuestos son una contribución (pago) en dinero o en especie de carácter obligatorio, con la que cooperamos para fortalecer la economía del país».

\title{
Concepto de cultura fiscal
}

Primero se abordará el término cultura, de Kroeber y Cluckhoholm (1952), citado por Reyes Rodríguez (2016), quienes indican que

\begin{abstract}
la cultura consiste en pautas de comportamiento, explícitas o implícitas, adquiridas y transmitidas mediante símbolos y constituye el patrimonio singularizador de los grupos humanos, incluida su plasmación en objetos; el núcleo esencial de la cultura son las ideas tradicionales (es decir, históricamente generadas y seleccionadas) y, especialmente, los valores vinculados a ellas; los sistemas de culturas pueden ser considerados, por un parte, como productos de la acción, y por otra, como elementos condicionantes de la acción futura (p. 196).
\end{abstract}

A continuación, se presenta el concepto de cultura fiscal, la cual «viene definida por el conjunto de ideas, valores y actitudes que inspiran y orientan la conducta de todos los actores sociales que intervienen en la configuración de la realidad fiscal de un país». (Goenaga Ruiz de Zuazu, 2012, p. 43). Para efectos de este estudio, se entiende por cultura fiscal el conjunto de creencias, actitudes y valores que tienen los contribuyentes, en este caso específico los trabajadores asalariados del estado de Yucatán, respecto al pago de impuestos, y cómo estas afectan la decisión de cumplir o no con sus obligaciones fiscales. En este sentido, pueden poseer una cultura fiscal que los predisponga a querer cumplir con estos pagos o una cultura fiscal adversa a dicho cumplimiento.

Para comprender mejor el concepto de cultura fiscal, antes se analizará cada una de estas variables. En primer lugar, las creencias son ideas aceptadas por la sociedad con las que el individuo se encuentra en su desarrollo y las cuales adopta como interpretación de la realidad, es decir: creer conlleva a la predisposición de actuar como si aquello en lo que se cree fuera verdadero, como si realmente existiera (Díez Patricio, 2017). En tanto que la actitud «es considerada como una disposición interna de carácter aprendido y duradera que sostiene las respuestas favorables o 
desfavorables del individuo hacia un objeto o una clase de objetos del mundo social» (Barriga Jiménez et al., 1998, p.118). Por último, los valores «pueden ser definidos como creencias prescriptivas duraderas sobre la preferencia de un modo de existencia o de conducta concreta en detrimento de otros modos de conducta y estados finales. La teoría sobre el sistema de creencias coloca los valores en el núcleo de la personalidad del individuo» (Palacios Navarro, 1997, p. 114).

\section{Estudios sobre cultura fiscal en otros países}

Los resultados de una investigación realizada en Australia acerca de las creencias y punto de vista de los contribuyentes, demostraron que las decisiones de éstos respecto al pago de sus impuestos, no son un simple cálculo de los costos y beneficios financieros o de cumplir o no con los requerimientos del sistema financiero. Por el contrario, éstos señalaron que las actitudes y las creencias que los contribuyentes tienen a cerca de la oficina de impuestos pueden afectar su cumplimiento (James et al., 2005).

Aunado a lo anterior, Reckers et al., (1994) señalan que las creencias morales individuales son determinantes en la decisión de cumplir con el pago de impuestos, pues cuando la evasión de estos se considera un asunto moral, los individuos son menos propensos a evadirlos, sin importar la situación fiscal en la que se encuentran. Por otro lado, las personas que no ven la evasión de impuestos como un asunto ético, son más propensas a no tributar.

\section{METODOLOGÍA}

La investigación realizada fue descriptiva, no experimental, transversal, de enfoque cuantitativo. Tomando en cuenta el tipo de estudio y los objetivos planteados, el método adecuado es el comparativo, ya que se tiene el interés de comparar el nivel de creencias, actitudes y valores que tienen un grupo de trabajadores encuestados, con respecto al pago de sus impuestos. Se hizo el análisis estratificado, de acuerdo con el salario mensual, para lo cual se elaboraron tablas de contingencia.

\section{Participantes}

Para calcular el tamaño de la muestra se tomó en cuenta los datos del Centro de Estudios de las Finanzas Públicas (CEFP, 2018), los cuales indican que la población asalariada en Yucatán rebasa los 100000 trabajadores, por lo que se considera una población infinita. Para ello, la fórmula empleada para el cálculo del tamaño de poblaciones infinitas fue:

$n=\left(3.84 * p^{*} q\right) / e^{2}$

Siendo $p=$ probabilidad de éxito, $q=$ probabilidad de fracaso y e=error de estimación.

Dónde: $p=0.50, q=0.50$ y e=6\%; al sustituir la fórmula se obtuvo un tamaño de muestra de 267 personas que serían encuestadas. Dado el objetivo de la investigación, se determinó que los encuestados debían ser asalariados de empresas privadas y dependencias del sector público. Los municipios seleccionados para aplicar la encuesta fueron Mérida, Umán y Tekax, por los siguientes 
motivos: Mérida es la capital y concentra la mayor población del Estado; Umán es uno de los tres municipios centrales de la zona metropolitana del Estado de Yucatán y se encuentra en conurbación continua a Mérida; por su parte, Tekax, es el municipio que se encuentra al sur del Estado y colinda con Campeche y Quintana Roo. Los encuestados fueron seleccionados de manera aleatoria, resultando 132 hombres, 129 mujeres y seis personas que no indicaron su sexo. Los escogidos fueron abordados en un hospital de Tekax, una empresa privada de la ciudad de Mérida, dos empresas privadas de la ciudad de Umán y entre trabajadores asalariados de la Universidad Tecnológica Metropolitana (UTM), aplicándose trescientos cuestionarios, los cuales fueron sometidos a limpieza, quedando 267 cuestionarios efectivos. El levantamiento de datos se realizó del 2 de mayo al 31 de julio de 2019.

\section{Procedimiento}

El estudio de investigación se realizó en dos etapas: en la primera etapa se definió la problemática a abordar y determinar si los trabajadores asalariados que prestan sus servicios a empresas públicas y privadas en las ciudades de Mérida, Umán y Tekax tienen cultura fiscal que los disponga a tributar de manera consciente, solidaria y responsable al Estado. Se eligió como técnica de recolección de información la encuesta, la cual se aplicó de manera autoadministrada a los sujetos de estudio.En la segunda etapa, se construyó un instrumento de recolección de información que en este caso fue un cuestionario dividido en cuatro secciones: i) la formulación de 24 preguntas sobre aspectos demográficos y socioeconómicos, ii) planteamiento dos preguntas que permitieran determinar las creencias de los encuestados respecto a la obligatoriedad de pagar impuestos, iii) esta sección consta de dos planteamientos que permiten conocer las actitudes de los sujetos de estudio respecto a su disposición para pagar impuestos y, iv) se registran dos enunciados que permiten saber los valores cívicos de los encuestados en su responsabilidad de pagar impuestos. En total son seis preguntas destinadas a recabar información sobre la cultura fiscal, distribuidas en tres variables: creencias, actitudes y valores; para estas se usó la escala Likert, se asignó el valor de 1 a la respuesta totalmente en desacuerdo, 2 en desacuerdo, 3 ni acuerdo ni en desacuerdo, 4 de acuerdo y 5 totalmente de acuerdo.

Para la validación del cuestionario se aplicó una prueba piloto a 33 trabajadores pertenecientes a la iniciativa privada; con los resultados obtenidos se creó una base de datos en el programa Excel y se realizó un análisis de fiabilidad mediante Alfa de Cronbach, dando como resultado lo que se muestra en la ecuación (1) y (2):

$$
\alpha=\frac{K}{K-1}\left[1-\frac{\sum V i}{V t}\right]
$$

Dónde:

$\alpha=$ Alfa de Cronbach

$K=$ (Número de ítems)

$\mathrm{Vi}=$ Varianza de cada ítem

$\mathrm{Vt}=$ Varianza total

$$
\alpha=\frac{6}{6-1}\left[1-\frac{7.038069796}{32.28282828}\right]=0.93838464
$$


Se consideró que el instrumento tiene un excelente nivel de fiabilidad. Además, el cuestionario fue validado por profesores expertos de la Facultad de Contaduría y Administración de la Universidad Autónoma de Yucatán (AUDY).

1. En la tercera etapa del estudio de investigación se determinó la muestra.

2. En la cuarta etapa del estudio de investigación se realizó el levantamiento de datos en campo.

\section{RESULTADOS}

En esta investigación se realizó un análisis estratificado, según el nivel de salario mensual y la cultura fiscal que tiene una muestra de trabajadores asalariados de tres municipios del estado de Yucatán, sobre la disposición a tributar de manera consciente, solidaria y responsable, basada en las variables de creencias, actitudes y valores.

Inicialmente se describe el perfil de la muestra, la cual está formada por 267 trabajadores, de los cuales 132 son de género masculino, 129 femenino y seis personas que no indicaron su sexo; la edad oscila entre los 18 y los 61 años, siendo la media 36 años. Con respecto al grado académico de los encuestados, el $36 \%$ tiene la educación básica completa, la cual, en México, comprende la primaria, la secundaria y el bachillerato; el $37 \%$ tiene educación universitaria completa, y el $27 \%$ estudios de posgrado. Con respecto al sueldo, la información se aprecia en la Tabla 1.

Tabla 1. Nivel de salario de los trabajadores encuestados Table 1. Salary level of surveyed employees

\begin{tabular}{|c|c|}
\hline Nivel salarial mensual & $\%$ \\
\hline a) Entre $\$ 3.000 .00$ y $\$ 4.999 .00$ & $22 \%$ \\
\hline b) Entre $\$ 5.000 .00$ y $\$ 9.999 .00$ & $25 \%$ \\
\hline c) Entre \$10 000.00 y \$ 14999.00 & $21 \%$ \\
\hline d) Entre $\$ 15000.00$ y \$19 999.00 & $18 \%$ \\
\hline e) Entre $\$ 20000.00$ y $\$ 24999.00$ & $7 \%$ \\
\hline \multirow[t]{2}{*}{ f) \$25000 o más } & $6 \%$ \\
\hline & $100 \%$ \\
\hline
\end{tabular}

Con respecto a la primera variable, se pretende conocer qué creen los encuestados con respecto al pago de impuestos; para ello se plantearon dos dimensiones, preguntándoseles: 1) Es responsabilidad de los ciudadanos pagar impuestos y 2) Los impuestos que se pagan al Estado sirven para costear servicios y obra pública. En la escala Likert se asignó el valor de 1 a la respuesta totalmente en desacuerdo; el valor 2, en desacuerdo; el 3, ni de acuerdo ni en desacuerdo; el valor, de 4 de acuerdo; y el 5, a totalmente de acuerdo. 
En la Figura 1 se muestran los resultados obtenidos. Allí, del total de los encuestados, el $69.3 \%$ está de acuerdo con la idea de que es responsabilidad de los ciudadanos pagar impuestos; sin embargo, al realizar el análisis por estrato, de acuerdo al salario mensual, se observa una diferencia de la percepción: menos de la mitad, es decir, el $46.6 \%$ de los trabajadores encuestados, con un salario mensual menor a \$4999, están de acuerdo o muy de acuerdo con el pago de impuestos; en tanto que el $88.2 \%$ de los trabajadores, con un ingreso superior a \$25000 mensual, están de acuerdo o muy de acuerdo.

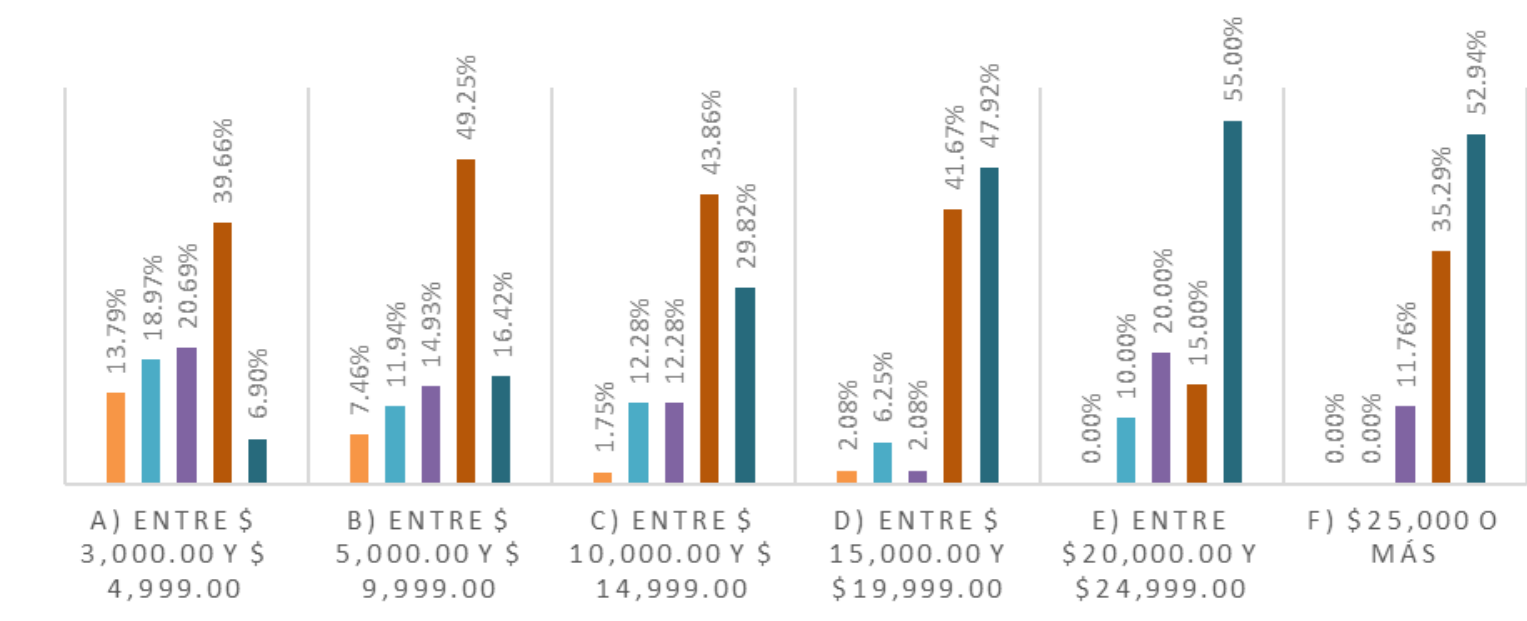

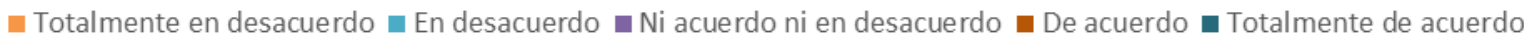

Figura 1. Es responsabilidad de los ciudadanos pagar impuestos

Figure 1. Citizens should pay taxes

Fuente: elaboración propia.

Con los datos anteriores se interpreta que, con respecto a la responsabilidad de los ciudadanos de pagar impuestos, la muestra tiene un promedio de 3.7, con una varianza de 1.3; los encuestados, con salarios mensuales de entre $\$ 3000$ y $\$ 4999$, tienen un promedio de 3.1; por el contrario, aquellos que tienen un ingreso superior a $\$ 15000$ mensuales, tienen un promedio superior a 4, y la dispersión de los datos, entre estos últimos, es menor.

De los datos presentados en la Tabla 2, se tiene que el 61.05\% de la muestra total está de acuerdo o muy de acuerdo con que los impuestos que se pagan al Estado sirven para costear servicios y obra pública; no obstante, analizando la información por estratos de salario mensual, se observa que el $50 \%$ de los encuestados, con ingresos menores a \$4999.00, está de acuerdo con esta creencia, mientras que el $82.35 \%$ de los encuestados, con un salario de $\$ 25000$ o más, está de acuerdo. 
Tabla 2. Los impuestos que se pagan al Estado sirven para costear servicios y obra pública Table 2. The taxes paid to the government are used to fund public services and works

\begin{tabular}{|c|c|c|c|c|c|c|}
\hline Salario mensual & $\begin{array}{c}\text { Totalmente } \\
\text { en } \\
\text { desacuerdo }\end{array}$ & $\begin{array}{c}\text { En } \\
\text { desacuerdo }\end{array}$ & $\begin{array}{c}\text { Ni de } \\
\text { acuerdo ni } \\
\text { en } \\
\text { desacuerdo }\end{array}$ & $\begin{array}{c}\text { De } \\
\text { acuerdo }\end{array}$ & $\begin{array}{l}\text { Totalmente } \\
\text { de acuerdo }\end{array}$ & $\begin{array}{r}\text { Total } \\
\text { genera }\end{array}$ \\
\hline a) Entre $\$ 3000$ y $\$ 4999$ & 7 & 7 & 15 & 26 & 3 & 58 \\
\hline b) Entre $\$ 5000.00$ y $\$ 9999$ & 7 & 12 & 14 & 21 & 13 & 67 \\
\hline c) Entre $\$ 10000.00$ y \$14 999 & 3 & 7 & 4 & 26 & 17 & 57 \\
\hline d) Entre \$15 000 y \$19 999 & 4 & 8 & 6 & 16 & 14 & 48 \\
\hline e) Entre $\$ 20000.00$ y $\$ 24999$ & 2 & 1 & 4 & 4 & 9 & 20 \\
\hline f) \$25000 o más & 1 & & 2 & 6 & 8 & 17 \\
\hline Total general & 24 & 35 & 45 & 99 & 64 & 267 \\
\hline
\end{tabular}

Los datos descritos en la Tabla 3 sobre la creencia de que los impuestos que se pagan al Estado sirven para costear servicios y obra pública (como se observa en los resultados) muestran que la media de la creencia es igual a 3.5, con una desviación estándar de 1.2; además, en esta variable el promedio en la aceptación de la creencia incrementa en los trabajadores con mayores ingresos.

Tabla 3. Datos descriptivos de la creencia de que los impuestos que se pagan al Estado sirven para costear servicios y obra pública

Table 3. Descriptive data of the belief that The taxes paid to the government are used to fund public services and works

\begin{tabular}{lccc}
\hline Salario mensual & Promedio de respuesta Likert & Varianza & \multicolumn{2}{c}{ Desviación estándar } \\
\hline a) Entre \$3000 y \$4999 & 3.2 & 1.2 & 1.1 \\
\hline b) Entre \$5000.00 y \$9999 & 3.3 & 1.6 & 1.3 \\
\hline c) Entre \$10 000 y \$14 999 & 3.8 & 1.3 & 1.2 \\
\hline d) Entre \$15 000 y \$19 999 & 3.6 & 1.7 & 1.3 \\
\hline e) Entre \$20 000 y \$24 999 & 3.9 & 1.8 & 1.3 \\
\hline f) \$25 000 o más & 4.2 & 1.2 & 1.1 \\
\hline Total & 3.5 & 1.5 & 1.2 \\
\hline
\end{tabular}

Con respecto a la segunda variable, también se plantearon dos dimensiones, con las cuales se pretende medir la actitud que tienen los trabajadores asalariados para pagar los impuestos: 1) Si tuviera la oportunidad como trabajador de elegir entre pagar o no pagar impuestos. Yo elegiría pagar mis impuestos, y 2) Si tuviera un negocio (empresa) pagaría puntualmente mis impuestos. Los resultados se observan en la Figura 2. 


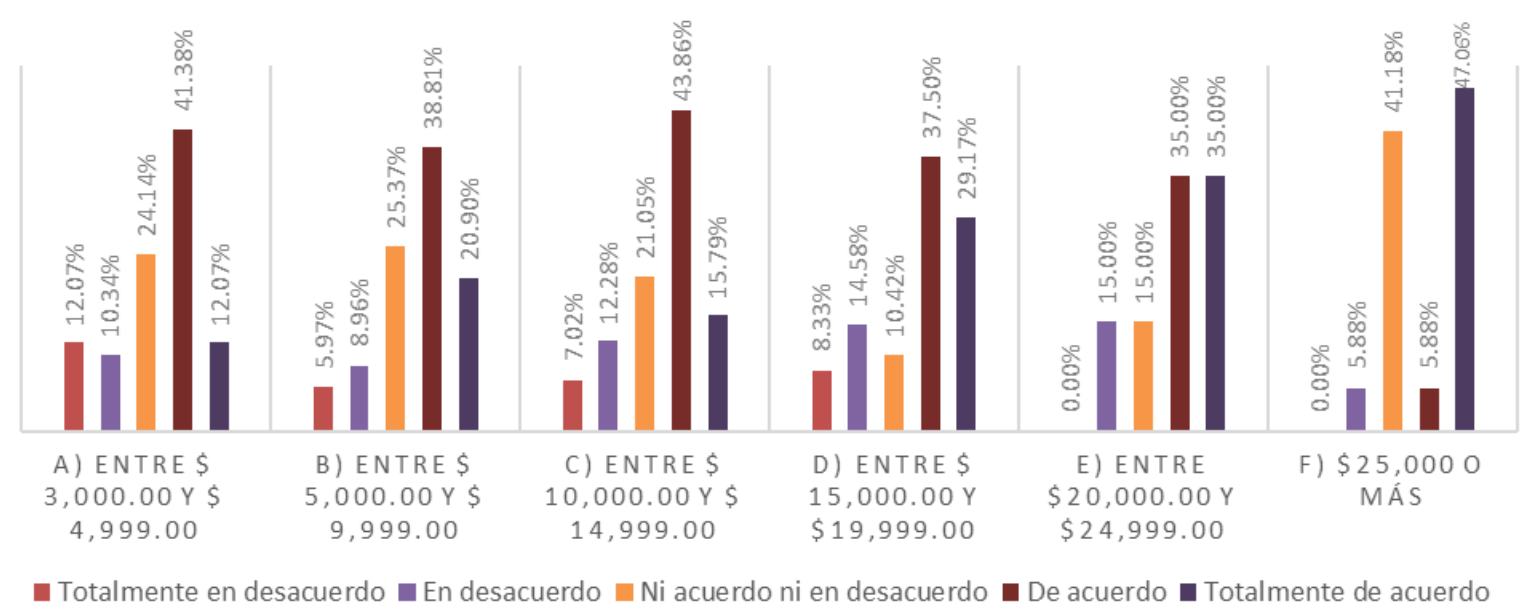

Figura 2. Si tuviera la oportunidad como trabajador de elegir entre pagar o no pagar impuestos. Yo elegiría pagar mis impuestos

Figure 2. If I, as an employee, could choose between paying taxes or not, I would pay my taxes Fuente: elaboración propia.

Con relación a la actitud que tienen los encuestados de pagar sus impuestos, se observa que el 59.9\% del total elegiría pagar sus impuestos; al realizar el análisis por estrato, no existe una diferencia significativa de la respuesta entre los niveles más bajos y más altos, dado que el $53.45 \%$ de los trabajadores encuestados, con ingresos entre $\$ 3000$ y $\$ 4999$, y el $52.94 \%$, con ingresos superiores a $\$ 25000$, elegirían pagar sus impuestos; sin embargo, los trabajadores con ingresos entre $\$ 15000$ y \$24 999 demuestran una actitud más dispuesta a la contribución de sus impuestos, ya que entre el $67 \%$ y $70 \%$ respondieron que elegirían pagar.

La muestra de trabajadores encuestados, como se observa en la Tabla 4, tiene un promedio de respuesta de 3.6, y una desviación estándar de 1.2 con respecto a la actitud que tendrían si tuvieran la oportunidad de elegir pagar o no pagar los impuestos; esto significa que la mayoría sí elegiría pagar. El promedio de respuesta con relación al importe de salario mensual devengado es muy parecido en todos los rangos. En la segunda dimensión se les hizo el planteamiento hipotético: si los encuestados tuvieran un negocio (empresa), ¿ pagarían puntualmente sus impuestos? Con ellos se pretende ver la actitud que tienen respecto del pago de impuestos al visualizarse como empresarios.

Tabla 4. Datos descriptivos de la actitud: si tuviera la oportunidad como trabajador de elegir entre pagar o no pagar impuestos. Yo elegiría pagar mis impuestos

Table 4. Descriptive data of the attitude that If I, as an employee, could choose between paying taxes or not, I would pay my taxes

\begin{tabular}{cccc}
\hline Salario mensual & Promedio de respuesta Likert & Varianza & Desviación estándar \\
\hline a) Entre $\$ 3000$ y $\$ 4999$ & 3.3 & 1.4 & 1.2 \\
\hline b) Entre $\$ 5000.00$ y \$9999 & 3.6 & 1.2 & 1.1 \\
\hline c) Entre $\$ 10000$ y \$14 999 & 3.5 & 1.3 & 1.1 \\
\hline d) Entre $\$ 15000$ y \$19 999 & 3.6 & 1.6 & 1.3 \\
\hline e) Entre $\$ 20000$ y \$24 999 & 3.9 & 1.1 & 1.1 \\
\hline f) \$25 000 o más & 3.9 & 1.2 & 1.1 \\
\hline & 3.6 & 1.3 & 1.2 \\
\hline
\end{tabular}

Fuente: elaboración propia. 
En la Figura 3, los resultados obtenidos indican que el $85.77 \%$ de la muestra tiene una actitud positiva ante este planteamiento hipotético, es decir, que pagarían puntualmente sus impuestos si tuvieran una empresa. Del análisis por estrato, se tiene que de los trabajadores encuestados con ingresos de entre $\$ 3000$ y $\$ 4999$, el $74.14 \%$ está de acuerdo o muy de acuerdo. Los porcentajes de aceptación se incrementan conforme se incrementa el salario mensual, siendo así que, un $95 \%$ de los trabajadores, con ingresos superiores a $\$ 25000$, están de acuerdo o muy de acuerdo.

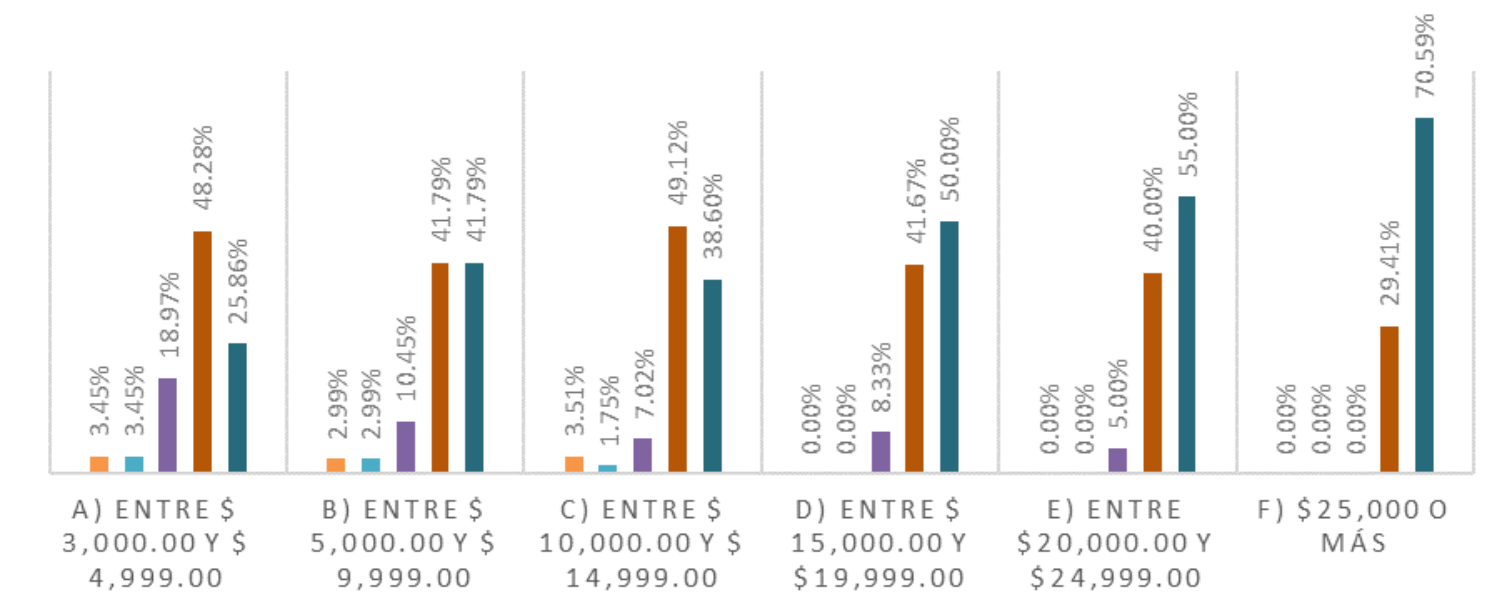

- Totalmente en desacuerdo $\square$ En desacuerdo $\square$ Ni acuerdo ni en desacuerdo $\square$ De acuerdo $\square$ Totalmente de acuerdo

Figura 3. Si tuviera un negocio (empresa). Pagaría puntualmente mis impuestos

Figure 3. If I had a business (company), I would pay my taxes on time Fuente: elaboración propia.

El promedio obtenido en las respuestas sobre Si tuviera un negocio (empresa). Pagaría puntualmente mis impuestos es el más alto de todas las variables analizadas; es decir, la actitud que tiene la muestra encuestada con relación al pago de impuestos bajo este planteamiento hipotético es muy positiva, pues la mayoría de los encuestados están de acuerdo o muy de acuerdo. Allí también se observa una desviación estándar de 0.9 .

Con relación a la variable de valores, se analizó la postura de los encuestados respecto de dos dimensiones, 1) El pago de impuestos es un acto cívico como ciudadano, y 2) Quien paga sus impuestos demuestra valores como la honradez, la responsabilidad y la solidaridad. Con ello se buscó verificar si los encuestados concuerdan con estos valores.

En la Figura 4 se observa que, de la muestra total, el 74.16\% de los encuestados están de acuerdo o muy de acuerdo con que el pago de impuestos, pues es un acto cívico como ciudadano. Asimismo, analizando las respuestas con relación al salario mensual, se observa que, en términos generales, los trabajadores con ingresos entre $\$ 3000$ y $\$ 4999$, es decir, el $60.34 \%$ dijeron estar de acuerdo o muy de acuerdo; en tanto que en los trabajadores con ingresos mayores a $\$ 25000$, la aceptación fue del $88.24 \%$. Lo anterior indica que, a mayor ingreso, mayor porcentaje de aceptación del pago de impuestos. 


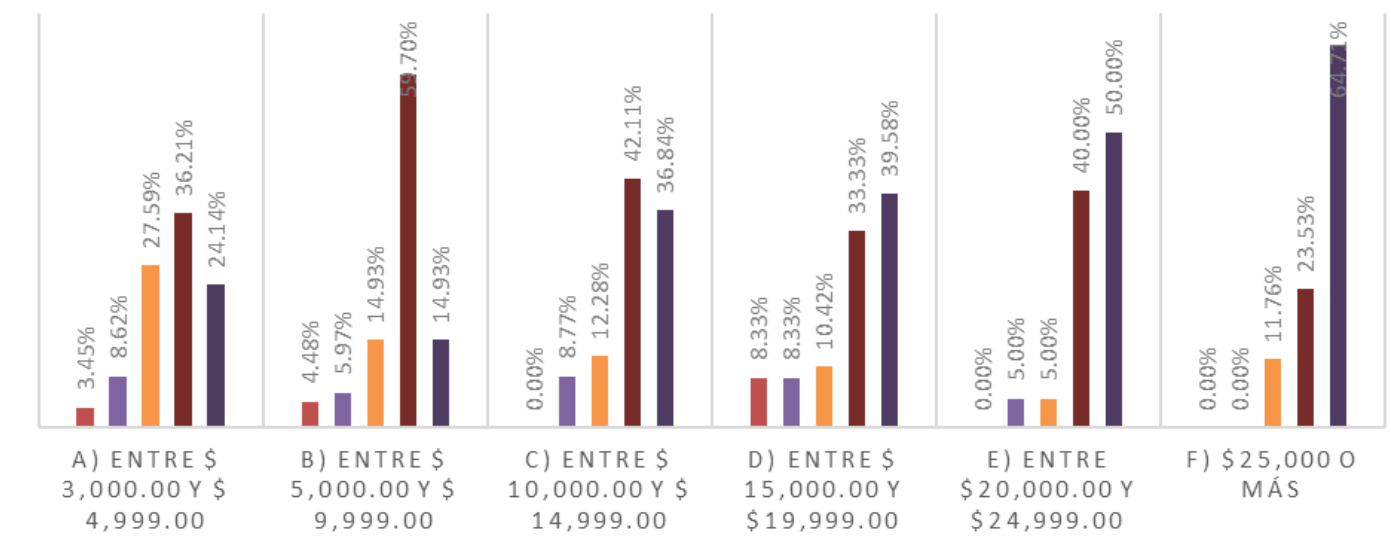

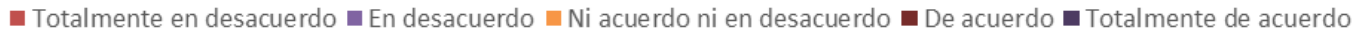

Figura 4. El pago de impuestos es un acto cívico como ciudadano

Figure 4. Paying taxes is a civic action as a citizen Fuente: elaboración propia.

Por lo que se refiere al valor, el pago de impuestos es un acto cívico como ciudadano. Se observa que la muestra respondió con un promedio de 3.9 y una desviación estándar de 1.1, lo que significa que la mayoría de los encuestados están de acuerdo con este valor; no obstante, al hacer el análisis por estrato se aprecia que la respuesta es más cercana a 5 conforme se eleva el salario mensual; asimismo, la desviación estándar disminuye en este rango de salario.

Por último, se les preguntó a los encuestados si están de acuerdo o no con la aseveración: quien paga sus impuestos demuestra valores como la honradez, la responsabilidad y la solidaridad. En la Figura 5 se observa que el $69.66 \%$ de la muestra está de acuerdo o totalmente de acuerdo con el enunciado Quien paga sus impuestos demuestra valores como la honradez, la responsabilidad y la solidaridad. Al analizar la variable, de acuerdo al estrato de salario mensual, se advierten diferencias en las respuestas de los encuestados, pues el porcentaje de aceptación aumenta conforme el nivel de salario. Así, los trabajadores con ingresos entre $\$ 3000$ y $\$ 4999$, están de acuerdo o muy de acuerdo, lo que representa un porcentaje de $60.34 \%$, y aquellos que tienen ingresos superiores a $\$ 25000$, tienen un porcentaje de $94.12 \%$.

Los resultados obtenidos detallan que el promedio de concordancia de la muestra con la aseveración Quien paga sus impuestos demuestra valores como la honradez, la responsabilidad y la solidaridad es del 3.9, con una desviación estándar de 1.1, por lo que se interpreta que la mayoría está de acuerdo. También se observa que el promedio se incrementa conforme aumenta el salario mensual y, por el contrario, la desviación estándar disminuye en este sentido, lo que indica que los asalariados con mayores ingresos, en su mayoría, están muy de acuerdo. 


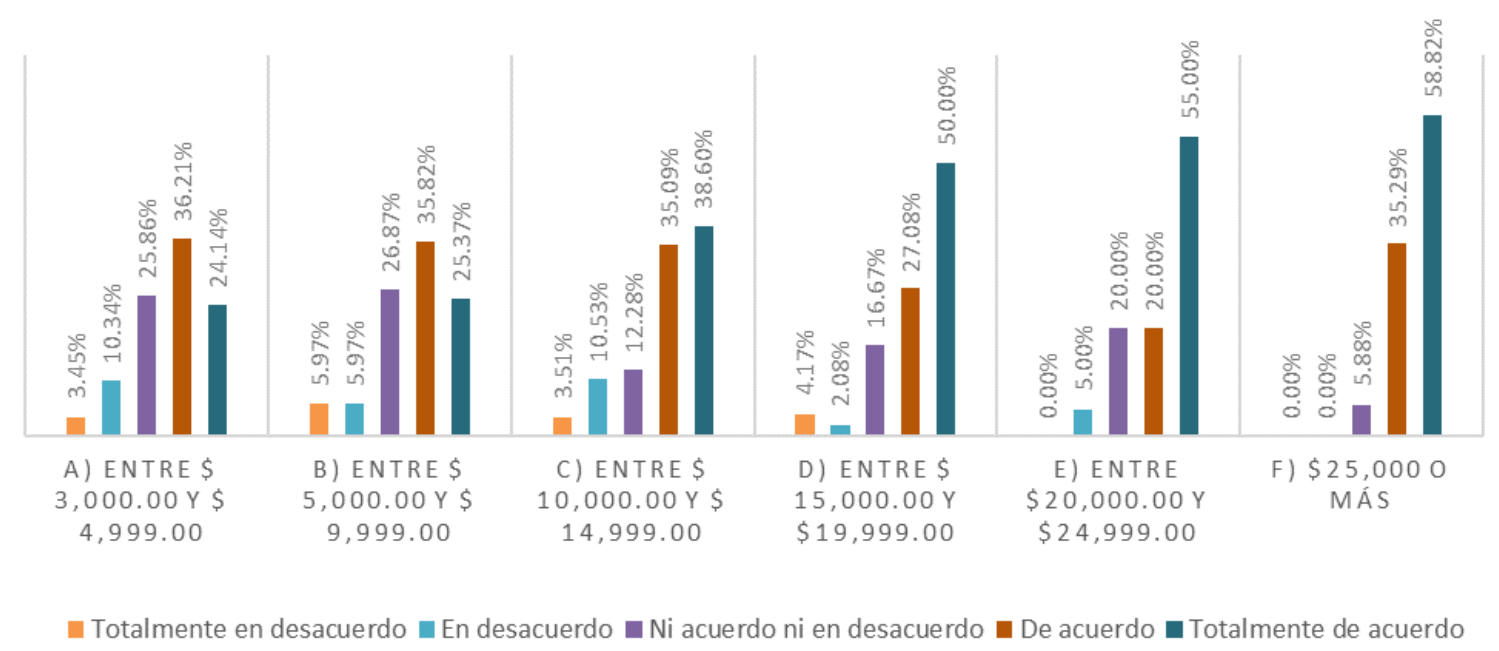

Figura 5. Quien paga sus impuestos demuestra valores como la honradez, la responsabilidad y la solidaridad Figure 5. Those who pay taxes demonstrate they have values such as honesty, responsibility, and solidarity Fuente: elaboración propia.

\section{DISCUSIÓN}

Los resultados difieren con un estudio realizado en Guanajuato sobre cultura fiscal, el cual estaba enfocado al público en general y a alumnos de primaria y secundaria, a quienes se les preguntó: si tuviera que escoger, ¿pagaría sus impuestos? El 55\% de la muestra del público en general respondió que no pagaría, mientras que solo el $45 \%$ lo haría (Andrade Oseguera \& García Vargas, 2012). Lo anterior contrasta lo respondido por el público en general encuestado en el estudio realizado por Andrade Oseguera y García Vargas, y las respuestas obtenidas de los trabajadores asalariados en esta investigación, en donde el 59.93\% contestó qué si tuvieran la oportunidad como trabajadores de elegir entre pagar o no impuestos, elegirían pagarlos.

Para Bushan y Medury (2013) la cultura fiscal tiene una relación positiva con la edad, lo que significa que se incrementa de acuerdo con el nivel académico y los ingresos. Esto concuerda con los hallazgos del presente estudio respecto de las variables de creencias y valores, pero difiere para la variable actitud; esto se aprecia con los siguientes resultados: en cuanto a la creencia de que es responsabilidad de los ciudadanos pagar impuestos, solo el $46.55 \%$ de los trabajadores, con ingresos de entre \$3000 y \$4999 mensuales, están de acuerdo o totalmente de acuerdo, mientras que el $88.24 \%$ de los trabajadores, con ingresos mayores a \$25 000 mensuales, están de acuerdo o totalmente de acuerdo. Con respecto a la creencia de que los impuestos que se pagan al Estado sirven para costear servicios y obra pública, se obtuvo que el $50 \%$ de los trabajadores, con ingresos de entre $\$ 3000$ y $\$ 4999$ mensuales, están de acuerdo o totalmente de acuerdo, en tanto que el $82.35 \%$ de los trabajadores, con ingresos mayores a \$25000 mensuales, están de acuerdo o totalmente de acuerdo. Con relación a la variable de valores, se encontró que el $60.34 \%$ de los trabajadores encuestados, con ingresos de entre $\$ 3000$ y $\$ 4999$ mensuales, consideran un acto cívico pagar impuestos, y el $88.24 \%$ de los trabajadores, con ingresos superiores a $\$ 25000$ mensuales, están de acuerdo o totalmente de acuerdo. Asimismo, el $60.34 \%$ de los trabajadores encuestados, con ingresos de entre $\$ 3000$ y $\$ 4999$ mensuales, están de acuerdo o totalmente de acuerdo con el hecho 
de que quien paga sus impuestos demuestra valores como la honradez, la responsabilidad y la solidaridad.

En relación con la variable actitud, se observó una respuesta diferenciada ante las dos dimensiones planteadas; por un lado, cuando se les preguntó si ellos tuvieran la oportunidad de elegir pagar o no sus impuestos como trabajadores asalariados, no hubo diferencia entre los encuestados de menor y mayor ingreso, en ambos casos los porcentajes de aceptación fueron a penas superiores al 50\%; en tanto que los trabajadores con sueldos de rangos medios entre los $\$ 15000$ y los $\$ 25000$ fueron los que demostraron una actitud de mayor compromiso con el pago de sus impuestos. Este comportamiento cambió al momento de hacer el planteamiento hipotético de si tuvieran un negocio ellos pagarían puntualmente sus impuestos, a lo que más del $85 \%$ respondió que están de acuerdo o muy de acuerdo; igualmente con esta dimensión se aprecia una relación entre el nivel salarial y la actitud de compromiso con el pago de los impuestos. Tomando en cuenta ambas dimensiones, esta variable contrasta con Bushan y Medury (2013).

\section{CONCLUSIONES}

Con los resultados obtenidos del análisis de las tres variables de la cultura fiscal, se concluye que la muestra de trabajadores encuestados sí poseen creencias, actitudes y valores que los dispone a tributar de manera consciente, solidaria y responsable. Sin embargo, al analizar los datos por estratos de salario mensual se observa una distinción en las respuestas, por lo tanto, hay una diferencia en la cultura fiscal de los trabajadores con ingresos menores con respecto a los trabajadores de mayor ingreso mensual: los segundos tienen una cultura fiscal más propensa al cumplimiento del pago de los impuestos en contraste con los primeros.

Con respecto a la variable creencias, se concluye que hay una relación positiva entre el nivel salarial y las creencias de que los impuestos son una obligación que la ciudadanía debe de pagar, al igual que los impuestos que se pagan al Estado sirven para costear servicios y obra pública. Se observó, además, que en la medida que los niveles de salario incrementan, incrementa también la aceptación de que los valores como la honradez, la responsabilidad la solidaridad y valores cívicos se demuestran pagando impuestos, por lo tanto, se concluye que hay una relación entre el nivel de salarios y los valores manifestados respecto al pago de impuestos. Por otro lado, las respuestas que se obtuvieron con las dimensiones relativas a la actitud fueron diferentes, de tal modo que no se puede decir que existe relación con el nivel de salario, por lo que se sugiere continuar con más estudios de esta variable para determinar posibles relaciones.

\section{CONFLICTOS DE INTERÉS}

Los autores declaran que no presentan conflictos de interés financiero, profesional o personal que pueda influir de forma inapropiada en los resultados obtenidos o las interpretaciones propuestas. 


\section{CONTRIBUCIÓN DE AUTORES}

Para el desarrollo de este proyecto todos los autores han realizado una contribución significativa especificada a continuación:

Elisa Cabrera-Ignacio: planteamiento de la problemática, marco teórico, metodología, discusión de resultados y conclusiones.

José Apolinar Zapata-Aguilar: planteamiento de la problemática, metodología y discusión de resultados.

\section{REFERENCIAS}

Abate, A. A. (2019). Factors affecting presumptive tax collection in Ethiopia: Evidence from category "C" taxpayers in Bahir Dar City. Journal of Tax Administration, v. 5, n. 2, 74-96. URL

Andrade Oseguera, M. A.; García Vargas, M. L. E. (2012). Cultura tributaria para una mayor recaudación fiscal. XVII Congreso Internacional de Contaduría, Administración e Informática. Universidad Nacional Autónoma de México. URL

Barriga Jiménez, S.; González Gabaldón, B.; Cantero Sánchez, F. J.; León Rubio, J. M.; Gómez Delgado, T.; Medina-Anzano, S. (1998). Psicología social: Orientaciones teóricas y ejercicios prácticos. Capítulo 2, tema 7. McGraw Hill. URL

Bushan, P.; Mendury, Y. (2013). Determining Tax Literacy of salaried Individuals-An Empirical Analysis. Journal of Business and Management (IOSR-JBM), v. 10, n. 6, 76-80. URL

Centro de Estudios de las Finanzas Públicas. (2018). Caracterización del Mercado Laboral en México. $\underline{U R L}$

Comisión Económica para América Latina y el Caribe. (2020). Estadísticas tributarias en América Latina y el Caribe 2020-México. URL

Díez Patricio, A. (2017). Más sobre la interpretación (II). Ideas y creencias. Revista de la Asociación Española de Neuropsiquiatría, v. 37, n. 131, 127-143. URL

Domingues, J. M.; González, C. Ch. (2013). Concepto de tributo: una perspectiva comparada BrasilEspaña. Revista Direito, v. 9, n. 2, 573-614. https://www.doi.org/10.1590/S1808-24322013000200009

Eagon, B. (2017). TIF-for-Tax: Upholding TIF's Original Purpose and Maximizing Its Use as a Catalyst for Community Economic Development. Wisconsin Law Review, v. 2017, n. 7, 179-224. URL 
Ermasova, N.; Haumann, C.; Burke, L. (2021). The relationship between culture and tax evasion across countries: Cases of the USA and Germany. International Journal of Public Administration, v. 44, n. 2, 115-131. https://doi.org/10.1080/01900692.2019.1672181

Goenaga Ruiz de Zuazu, M. (2012). Elementos principales de la cultura fiscal en América Latina. Documento de trabajo IELAT, n. 42, 40-59. URL

Huang, S. H.; Yu, M. M.; Hwang, M. S.; Wei, Y. S.; Chen, M. H. (2017). Efficiency of tax collection and tax management in Taiwan's local tax offices. Pacific Economic Review, v. 22, n. 4, 620-648. https://doi.org/10.1111/1468-0106.12235

James, S.; Murphy, K.; Reinhart, M. (2005). Taypayer Belief and Views: A New Survey. Australian Tax Forum, v. 20, n. 2, 157-188. URL

Lara Dorantes, R. (2009). La recaudación tributaria en México. Revista del Instituto de Ciencias Jurídicas de Puebla A. C., n. 23, 113-143. URL

Ley de Ingresos de la Federación para el ejercicio fiscal. (2018, 28 de diciembre). URL

Nerré, B. (Agosto de 2001). The emergence of a tax culture in Russia. University of Hamburg Germany. 57th Congress of the International Institute of Public Finance: The Role of Political Economy in the Theory and Practice of Public Finance. Congreso llevado a cabo en Linz, Austria. URL

Organización para la Cooperación y el Desarrollo Económicos. (s.f.). Base de datos global de estadísticas tributarias. Tax policy análisis. $\underline{U R L}$

Palacios Navarro, S. (1997). Los valores humanos y la comprensión de la desobediencia civil. Revista de Psicodidáctica, n. 3, 113-131. URL

Reckers, P. M. J.; Sanders, D. L; Roark, S. J. (1994). The influence of ethical attitudes on taxpayer Compliance. National Tax Journal, v. 47, n. 4, 825-836. https://doi.org/10.1086/NTJ41789111

Reyes Rodríguez, Y. (2016). Un estudio de enfoques y conceptos de cultura y su relación con la noción de identidad.Didasc@lia: Didáctica y Educación, v. 7, n. 4.195-206. URL

Richardson, G. (2008). The relationship between culture and tax evasion across countries: Additional evidence and extensions. Journal of International Accounting, Auditing and Taxation, v. 17, n. 2, 67-78. https://doi.org/10.1016/j.intaccaudtax.2008.07.002

Sistema de información legislativa. (s.f.). Ley de ingresos. URL 